\title{
Physical observables from boundary artifacts: scalar glueball in Yang-Mills theory
}

\author{
Abhishek Chowdhury, ${ }^{a}$ A. Harindranath ${ }^{b}$ and Jyotirmoy Maiti ${ }^{c}$ \\ ${ }^{a}$ Department of Physics, Raja Narendra Lal Khan Women's College, \\ Gope Palace, P.O. Vidyasagar University, Paschim Medinipur District, 721102, India \\ ${ }^{b}$ Theory Division, Saha Institute of Nuclear Physics, \\ 1/AF Bidhan Nagar, Kolkata 700064, India \\ ${ }^{c}$ Department of Physics, Barasat Government College, \\ $10 \mathrm{KNC}$ Road, Barasat, Kolkata 700124, India \\ E-mail: abhi109@gmail.com, a.harindranath@saha.ac.in, \\ jyotirmoy.maiti@gmail.com
}

ABSTRACT: By relating the functional averages of a generic scalar operator in simulations with Open (O) and Periodic (P) boundary conditions (BCs) respectively for SU(3) lattice gauge theory, we show that the scalar glueball mass and the glueball to vacuum matrix element can be extracted very efficiently from the former. Numerical results are compared with those extracted from the two point function of the time slice energy density (both $\mathrm{PBC}$ and $\mathrm{OBC}$ ). The scaling properties of the mass and the matrix element are studied with the help of Wilson (gradient) flow.

KeYwords: Lattice QCD, Lattice Gauge Field Theories, Lattice Quantum Field Theory

ARXIV EPRINT: 1509.07959 


\section{Contents}

1 Motivation 1

2 Relation between correlation functions in OBC and PBC 2

3 Determination of the mass and the matrix element 4

3.1 Periodic Boundary Condition (PBC) 4

3.2 Open Boundary Condition (OBC) 5

4 Numerical results $\quad 5$

A Connection between matrix elements 12

\section{Motivation}

In confronting experimental data, lattice Quantum Chromodynamics has achieved remarkable progress over the years. Nevertheless certain problems emerge as the continuum limit is approached, a major difficulty being the spanning of gauge configurations over different topological sectors when periodic boundary condition (PBC) is used in the temporal direction. To overcome this problem, open boundary condition (OBC) in the temporal direction has been proposed recently [1-3]. In order to avoid undesirable effects in the spectrum of the Hamiltonian, boundary conditions are retained to be periodic for the three-dimensional space, which ensures that the transfer matrix is unaltered. Earlier, the advantage of OBC over PBC has been recognized and profitably utilized in Density Matrix Renormalization Group calculations [4] applied to condensed matter systems. Later, some undesirable features of OBC for systems that do not possess an energy gap have been recognized and investigated in detail [5]. However, the systems under our consideration, namely, pure Yang-Mills theory and QCD fortuitously possess mass gaps. Moreover, OBC yields some unexpected extra dividends as we demonstrate in this work. For example, by studying the boundary artifacts in the vacuum expectation value of a one point function, one can extract the mass and operator matrix elements which are usually extracted from a two point function. We illustrate this idea in the context of the calculation of scalar glueball mass and glueball to vacuum matrix element in SU(3) Lattice Yang-Mills theory.

For extracting the mass and the matrix element in lattice Yang-Mills theory, smoothing of gauge fields is essential. The Wilson (gradient) flow [6-8] provides a very convenient tool for smoothing, with a rigorous mathematical underpinning. Unlike the conventional smearing techniques, the Wilson flow provides a common reference scale. Thus by choosing a particular flow time, one can study the scaling properties of observables extracted from lattice calculations employing different lattice spacings. It is interesting to perform such 
scaling studies for the glueball mass and the glueball to vacuum matrix element. Another noise reduction technique was recently investigated [9] in the extraction of glueball masses.

\section{Relation between correlation functions in $\mathrm{OBC}$ and $\mathrm{PBC}$}

We start from the standard Wilson action for SU(3) lattice gauge theory on a $L^{3} \times T$ lattice with periodic boundary conditions in all directions

$$
S_{\mathrm{PBC}}=\frac{2}{g^{2}} \sum_{x} \sum_{\mu<\nu} \operatorname{tr}\left[1-\operatorname{Re} U_{\mu \nu}(x)\right]
$$

where $U_{\mu \nu}(x)$ denotes the product of the link variables around a plaquette $P$ in the $\mu-\nu$ plane whose lower left hand corner is at $x$ and the sum is over all oriented plaquettes on the lattice.

Using transfer matrix arguments $[2,10,11]$, keeping in mind that with open boundary condition in the temporal direction, there are no temporal links connecting the time slice $x_{0}=T-1$ to the time slice $x_{0}=0$, one arrives at the action for $\mathrm{SU}(3)$ lattice gauge theory with open boundary condition

$$
S_{\mathrm{OPEN}}=\frac{2}{g^{2}} \sum_{x} \sum_{\mu<\nu} w(P) \operatorname{tr}\left[1-\operatorname{Re} U_{\mu \nu}(x)\right]
$$

where $w(P)$ is equal to 1 except for the spatial plaquette at time $x_{0}=0$ and $T-1$ which have weight $\frac{1}{2}$ and here the sum runs over the plaquettes having their corners within the time interval $[0, T-1]$.

Thus we find that $S_{\mathrm{PBC}}=S_{\mathrm{OPEN}}+\Delta S$ where

$$
\begin{aligned}
\Delta S=\frac{2}{g^{2}} \sum_{\mathbf{x}}\{ & \frac{1}{2} \sum_{i<j}\left(\operatorname{tr}\left[1-\operatorname{Re} U_{i j}(\mathbf{x}, T-1)\right]+\operatorname{tr}\left[1-\operatorname{Re} U_{i j}(\mathbf{x}, 0)\right]\right) \\
& \left.+\sum_{i} \operatorname{tr}\left[1-\operatorname{Re} U_{i 4}(\mathbf{x}, T-1)\right]\right\}
\end{aligned}
$$

Note that $e^{-\Delta S}$ is the transfer matrix element [10] between the time slices $T-1$ and 0 . Denoting the general transfer matrix element between time slices $x_{0}$ and $x_{0}+1$ by $e^{-L^{3} H_{m}\left(x_{0}\right)}$, we have

$$
\begin{gathered}
H_{m}\left(x_{0}\right)=\frac{2}{g^{2}} \frac{1}{L^{3}} \sum_{\mathbf{x}}\left\{\frac{1}{2} \sum_{i<j}\left(\operatorname{tr}\left[1-\operatorname{Re} U_{i j}\left(\mathbf{x}, x_{0}\right)\right]+\operatorname{tr}\left[1-\operatorname{Re} U_{i j}\left(\mathbf{x}, x_{0}+1\right)\right]\right)\right. \\
\left.+\sum_{i} \operatorname{tr}\left[1-\operatorname{Re} U_{i 4}\left(\mathbf{x}, x_{0}\right)\right]\right\} .
\end{gathered}
$$

An observable which we have extensively studied in our previous work [14] is the time slice energy density $\bar{E}\left(x_{0}\right)$ which was used as an interpolating operator to calculate the 
scalar glueball mass. We adopt a symmetric definition of the time slice energy density as

$$
\begin{aligned}
\bar{E}\left(x_{0}\right)=\frac{2}{g^{2}} \frac{1}{L^{3}} \sum_{\mathbf{x}}\left\{\sum_{i<j}\right. & \operatorname{tr}\left[1-\operatorname{Re} U_{i j}\left(\mathbf{x}, x_{0}\right)\right] \\
& \left.+\frac{1}{2} \sum_{i}\left(\operatorname{tr}\left[1-\operatorname{Re} U_{i 4}\left(\mathbf{x}, x_{0}\right)\right]+\operatorname{tr}\left[1-\operatorname{Re} U_{i 4}\left(\mathbf{x}, x_{0}-1\right)\right]\right)\right\}
\end{aligned}
$$

where $U_{\mu \nu}\left(\mathbf{x}, x_{0}\right)$ denotes the oriented plaquette in the $\mu-\nu$ plane with $\left(\mathbf{x}, x_{0}\right)$ at its lowerleft corner. The time slice $x_{0}$ is arbitrary for periodic boundary condition but it is restricted within the bulk when open boundary condition is imposed in the temporal direction. For the latter case, the definitions of $\bar{E}\left(x_{0}\right)$ with $x_{0}$ lying on the boundaries are given by

$$
\begin{aligned}
\bar{E}\left(x_{0}=0\right)=\frac{2}{g^{2}} \frac{1}{L^{3}} \sum_{\mathbf{x}} \frac{1}{2}\left\{\sum_{i<j}\right. & \operatorname{tr}\left[1-\operatorname{Re} U_{i j}(\mathbf{x}, 0)\right] \\
& \left.+\sum_{i} \operatorname{tr}\left[1-\operatorname{Re} U_{i 4}(\mathbf{x}, 0)\right]\right\}
\end{aligned}
$$

and $\bar{E}\left(x_{0}=T-1\right)=\frac{2}{g^{2}} \frac{1}{L^{3}} \sum_{\mathbf{x}} \frac{1}{2}\left\{\sum_{i<j} \operatorname{tr}\left[1-\operatorname{Re} U_{i j}(\mathbf{x}, T-1)\right]\right.$

$$
\left.+\sum_{i} \operatorname{tr}\left[1-\operatorname{Re} U_{i 4}(\mathbf{x}, T-2)\right]\right\} \text {. }
$$

To find a relation between $\left\langle\mathcal{O}\left(x_{0}\right)\right\rangle_{\mathrm{OPEN}}$ and $\left\langle\mathcal{O}\left(x_{0}\right)\right\rangle_{\mathrm{PBC}}$ where $\mathcal{O}$ is a generic scalar operator we start from

$$
\langle\mathcal{O}\rangle_{\mathrm{OPEN}}=\frac{\int \mathcal{D} U^{\prime} \mathcal{O}\left(x_{0}\right) e^{-S_{\mathrm{OPEN}}}}{\int \mathcal{D} U^{\prime} e^{-S_{\mathrm{OPEN}}}}
$$

where the measure $\mathcal{D} U^{\prime}$ excludes the measures for the links connecting the boundary time slices $\left(x_{0}=T-1\right.$ and $\left.x_{0}=0\right)$. However, as the integrands in both numerator and denominator do not depend on these links, we can include their measures without altering the result. This leads us to replace $\mathcal{D} U^{\prime}$ by $\mathcal{D} U$ which is the measure in case of PBC. Thus

$$
\left\langle\mathcal{O}\left(x_{0}\right)\right\rangle_{\mathrm{OPEN}}=\frac{\int \mathcal{D} U \mathcal{O}\left(x_{0}\right) e^{-S_{\mathrm{OPEN}}}}{\int \mathcal{D} U e^{-S_{\mathrm{OPEN}}}}=\frac{\int \mathcal{D} U \mathcal{O}\left(x_{0}\right) e^{-S_{\mathrm{PBC}}+\Delta S}}{\int \mathcal{D} U e^{-S_{\mathrm{PBC}}+\Delta S}}
$$

where the exponents of the integrands in both numerator and denominator on the right hand side consist of fields on a periodic lattice. Thus

$$
\begin{aligned}
\left\langle\mathcal{O}\left(x_{0}\right)\right\rangle_{\mathrm{OPEN}} & =\frac{\int \mathcal{D} U \mathcal{O}\left(x_{0}\right) e^{-S_{\mathrm{PBC}}+\Delta S} / \int \mathcal{D} U e^{-S_{\mathrm{PBC}}}}{\int \mathcal{D} U e^{-S_{\mathrm{PBC}}+\Delta S} / \int \mathcal{D} U e^{-S_{\mathrm{PBC}}}} \\
& =\left\langle\mathcal{O}\left(x_{0}\right)\right\rangle_{\mathrm{PBC}}+\frac{\left\langle\mathcal{O}\left(x_{0}\right) e^{\Delta S}\right\rangle_{\mathrm{PBC}}^{\text {connected }}}{\left\langle e^{\Delta S}\right\rangle_{\mathrm{PBC}}} \\
& =\left\langle\mathcal{O}\left(x_{0}\right)\right\rangle_{\mathrm{PBC}}+\frac{1}{r}\left\langle\mathcal{O}\left(x_{0}\right) e^{L^{3} H_{m}(T-1)}\right\rangle_{\mathrm{PBC}}^{\text {connected }}
\end{aligned}
$$

where $r=\left\langle e^{\Delta S}\right\rangle_{\mathrm{PBC}}=\left\langle e^{L^{3} H_{m}(T-1)}\right\rangle_{\mathrm{PBC}}$. 
As $e^{L^{3} H_{m}\left(x_{0}\right)}$ is also a scalar operator, from eq. (2.11) we have

$$
\left\langle\mathcal{O}\left(x_{0}\right)\right\rangle_{\mathrm{OPEN}} \approx\left\langle\mathcal{O}\left(x_{0}\right)\right\rangle_{\mathrm{PBC}}+2 C_{1}^{\prime} e^{-m T / 2} \cosh m\left(\frac{T}{2}-1-x_{0}\right) .
$$

where $m$ is the scalar glueball mass.

In comparison, the two point function for the time slice energy density in the case of PBC behaves as

$$
\left\langle\bar{E}\left(x_{0}\right) \bar{E}\left(x_{0}=0\right)\right\rangle_{\mathrm{PBC}} \approx C_{0}+2 C_{1} e^{-m T / 2} \cosh m\left(\frac{T}{2}-x_{0}\right) .
$$

where

$$
C_{1}=\frac{|\langle 0|E(0)| G\rangle|^{2}}{2 m}=\frac{C^{2}}{2 m} .
$$

Thus we find that one can extract certain two-point correlators by analyzing the data for the functional average of a scalar operator computed with open boundary (in the temporal direction) in the region of $x_{0}$ where it differs, due to the breaking of translational invariance, from the same computed with periodic boundary. Same technique can, in principle, be used to compute any n-point correlator in the scalar channel. In the case of lattice QCD with $\mathrm{OBC}$, same technique can also be used to extract the mass of the lowest two-pion state. A recent simulation with $2+1$ flavors [16] however encountered large scaling violations which unfortunately made such an extraction not possible.

We note that while the extraction of the glueball mass from the one point function is as straightforward as from the two point function, the extraction of the glueball to vacuum matrix element from the former is not as straightforward as from the latter. However for the operators $\left(\bar{E}\right.$ and $\left.H_{m}\right)$ used in this work, this becomes possible in the region of very small lattice spacing. See the appendix for the discussion. This is supported by our numerical results.

\section{Determination of the mass and the matrix element}

In this section, we discuss the methods for extraction of the glueball mass and the glueball to vacuum matrix element from the two-point (PBC and $\mathrm{OBC}$ ) and the one point $(\mathrm{OBC})$ correlation functions.

\subsection{Periodic Boundary Condition (PBC)}

In the case of PBC, the mass and the matrix element have to be extracted from the two point correlator. Because of the periodicity of the lattice we have

$$
\left\langle\bar{E}\left(x_{0}\right) \bar{E}\left(x_{0}=0\right)\right\rangle_{\mathrm{PBC}}=G\left(x_{0}\right) \simeq C_{0}+C_{1}\left[e^{-m x_{0}}+e^{-m\left(T-x_{0}\right)}\right] .
$$

The effective mass is calculated by solving the equation $F(m)=0$

$$
F=\left(r_{1}-1\right)[\cosh m(d t-1)-\cosh m d t]+\left(1-r_{2}\right)[\cosh m(d t+1)-\cosh m d t]
$$


where

$$
r_{1}=\frac{G\left(x_{0}-1\right)}{G\left(x_{0}\right)}, \quad r_{2}=\frac{G\left(x_{0}+1\right)}{G\left(x_{0}\right)} \quad \text { and } \quad d t=T / 2-x_{0} .
$$

The coefficient has been extracted as

$$
C_{1}=\frac{1}{2}\left(C_{1}^{(1)}+C_{1}^{(2)}\right)
$$

where

$$
\begin{aligned}
C_{1}^{(1)} & =\frac{G\left(x_{0}\right)-G\left(x_{0}+1\right)}{e^{-m x_{0}}+e^{-m\left(T-x_{0}\right)}-e^{-m\left(x_{0}+1\right)}-e^{-m\left(T-x_{0}-1\right)}} \\
\text { and } \quad C_{1}^{(2)} & =\frac{G\left(x_{0}-1\right)-G\left(x_{0}\right)}{e^{-m\left(x_{0}-1\right)}+e^{-m\left(T-x_{0}+1\right)}-e^{-m\left(x_{0}\right)}-e^{-m\left(T-x_{0}\right)}} .
\end{aligned}
$$

\subsection{Open Boundary Condition (OBC)}

In the case of $\mathrm{OBC}$, the mass and the matrix element can be extracted from the one point correlator as well as from the two point function as discussed in the previous section. In the case of two point correlator, because of the lack of translational invariance, we have

$$
\left\langle\bar{E}\left(x_{0}+x_{0}^{\prime}\right) \bar{E}\left(x_{0}^{\prime}\right)\right\rangle_{\mathrm{OBC}}=G\left(x_{0}\right) \simeq C_{0}+C_{1} e^{-m x_{0}}
$$

where $x_{0}^{\prime}$ is well within the bulk. Thus the effective mass is given by

$$
m=\ln \frac{G\left(x_{0}-1\right)-G\left(x_{0}\right)}{G\left(x_{0}\right)-G\left(x_{0}+1\right)} .
$$

We extract the coefficient as

$$
C_{1}=\frac{1}{2}\left(C_{1}^{(1)}+C_{1}^{(2)}\right)
$$

where

$$
C_{1}^{(1)}=\frac{G\left(x_{0}\right)-G\left(x_{0}+1\right)}{e^{-m x_{0}}-e^{-m\left(x_{0}+1\right)}} \quad \text { and } \quad C_{1}^{(2)}=\frac{G\left(x_{0}-1\right)-G\left(x_{0}\right)}{e^{-m\left(x_{0}-1\right)}-e^{-m x_{0}}}
$$

\section{Numerical results}

SU(3) gauge configurations in lattice Yang-Mills theory are generated with open boundary condition (denoted by $\mathrm{O}$ ) at different lattice volumes (by lattice volume we mean total number of lattice points) and gauge couplings using the openQCD program [12]. For comparison purposes, by implementing periodic boundary condition in temporal direction in the openQCD package, we have also generated gauge configurations (denoted by $\mathrm{P}$ ) for several of the same lattice parameters. Simulation details are given in table 1 . The parameter $t_{0}$ is defined in the context of Wilson flow [6-8] which is used to smooth the gauge configurations. The implicit equation

$$
\left\{t^{2}\langle\bar{E}(T / 2)\rangle\right\}_{t=t_{0}}=0.3
$$




\begin{tabular}{|c|l|l|l|l|l|l|}
\hline Lattice & Volume & $\beta$ & $N_{\text {cnfg }}$ & $\tau$ & $a[\mathrm{fm}]$ & $t_{0} / a^{2}$ \\
\hline$O_{1}$ & $24^{3} \times 48$ & 6.21 & 3970 & 3 & $0.0667(5)$ & $6.207(15)$ \\
\hline$O_{2}$ & $32^{3} \times 64$ & 6.42 & 3028 & 4 & $0.0500(4)$ & $11.228(31)$ \\
\hline$O_{3}$ & $48^{3} \times 96$ & 6.59 & 2333 & 5 & $0.0402(3)$ & $17.630(53)$ \\
\hline$O_{4}$ & $64^{3} \times 128$ & 6.71 & 181 & 10 & $0.0345(4)$ & $24.279(227)$ \\
\hline$P_{1}$ & $24^{3} \times 48$ & 6.21 & 3500 & 3 & $0.0667(5)$ & $6.197(15)$ \\
\hline$P_{2}$ & $32^{3} \times 64$ & 6.42 & 1958 & 4 & $0.0500(4)$ & $11.270(38)$ \\
\hline$P_{3}$ & $48^{3} \times 96$ & 6.59 & 295 & 5 & $0.0402(3)$ & $18.048(152)$ \\
\hline
\end{tabular}

Table 1. $N_{\text {cnfg }}$ is the number of configurations, $\tau$ is the trajectory length used in the HMC simulation and $t_{0} / a^{2}$ is the dimensionless reference Wilson flow time. $O$ and $P$ refer to ensembles with open and periodic boundary conditions in the temporal direction.

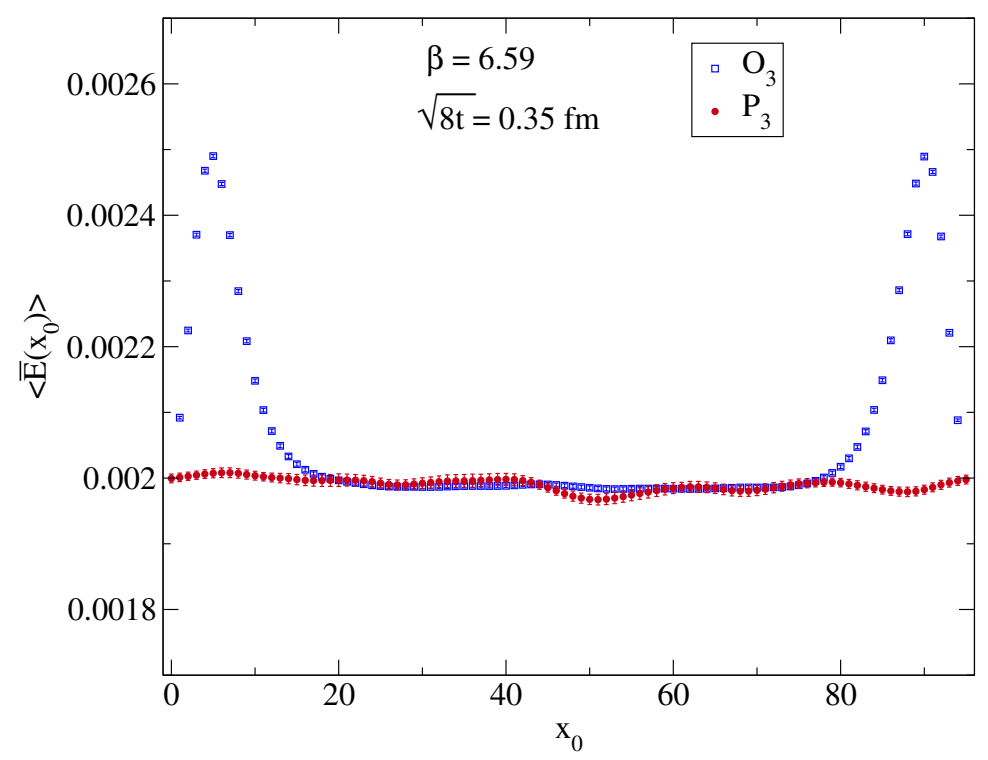

Figure 1. Plot of $\left\langle\bar{E}\left(x_{0}\right)\right\rangle$ versus $x_{0}$ at flow time $t=t_{0}$ at $\beta=6.59$ and lattice volume $48^{3} \times 96$ for ensemble $\mathrm{O}_{3}$ (filled circle) and ensemble $P_{3}$ (filled square).

with $t$ and $T$ being respectively the Wilson flow time and the temporal extent of the lattice, defines a reference flow time $t_{0}$ which provides a reference scale to extract physical quantities from lattice calculations. The effectiveness of the Wilson flow in the extraction of topological susceptibility [13], glueball mass [14] and topological charge density correlator [15] has been demonstrated recently.

In figure 1 we plot the configuration average of the time slice energy density averaged over the spatial volume $\left\langle\bar{E}\left(x_{0}\right)\right\rangle$ versus the time slice $x_{0}$ at the reference flow time $t=t_{0}[7]$ at $\beta=6.59$ and lattice volume $48^{3} \times 96$ for ensemble $O_{3}$ (filled circle) and ensemble $P_{3}$ (filled square). We find that in the boundary region at $x_{0}=0,\left\langle\bar{E}\left(x_{0}\right)\right\rangle_{\mathrm{OBC}}$ rises above and then decays to $\left\langle\bar{E}\left(x_{0}\right)\right\rangle_{\mathrm{PBC}}$. Similar behaviour is found in the boundary region $x_{0}=T-1$. 

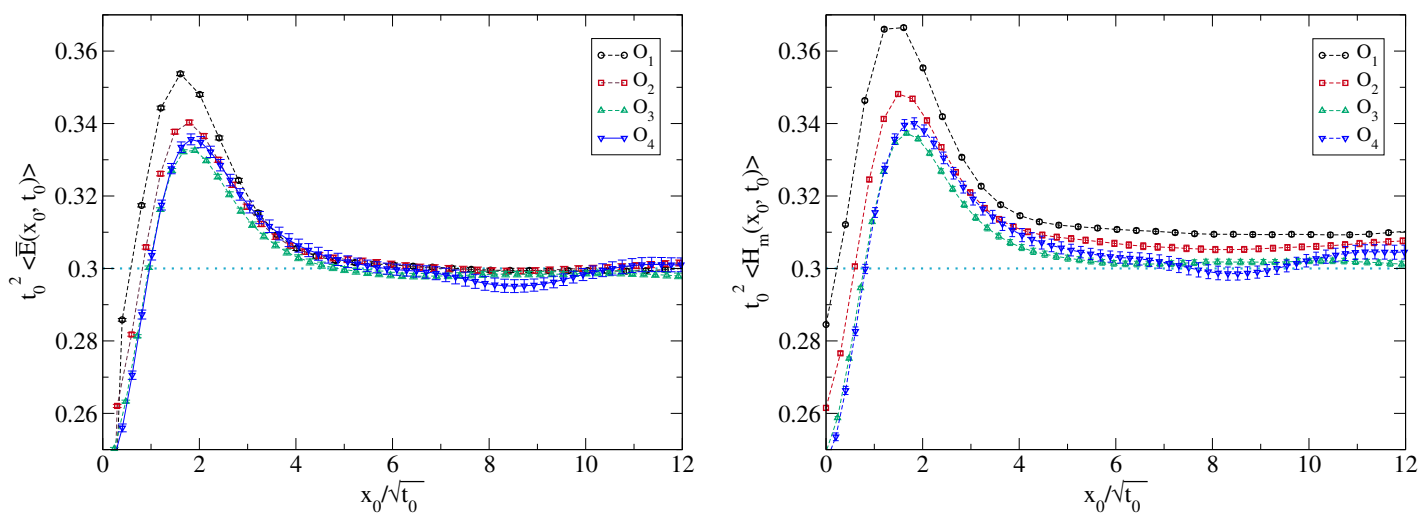

Figure 2. Plot of $t_{0}^{2}\left\langle\bar{E}\left(x_{0}, t_{0}\right)\right\rangle$ (left) and $t_{0}^{2}\left\langle H_{m}\left(x_{0}, t_{0}\right)\right\rangle$ (right) versus $x_{0} / \sqrt{t_{0}}$ for the ensembles $\mathrm{O}_{1}, \mathrm{O}_{2}, \mathrm{O}_{3}$ and $\mathrm{O}_{4}$.

As explained in section 2, the decay rate is determined by the mass of the lowest excitation in the scalar channel, namely the glueball mass.

In order to reliably determine the glueball mass from the decay of $\left\langle\bar{E}\left(x_{0}\right)\right\rangle_{\mathrm{OBC}}$ and $\left\langle H_{m}\left(x_{0}\right)\right\rangle_{\mathrm{OBC}}$ first we need to verify their scaling behaviour. Towards this goal, in figure 2 (left) we plot $t_{0}^{2}\left\langle\bar{E}\left(x_{0}, t_{0}\right)\right\rangle$ versus $x_{0} / \sqrt{t_{0}}$ for the ensembles $O_{1}, O_{2}, O_{3}$ and $O_{4}$. We note that, except for the largest lattice spacing, the data exhibit excellent scaling behaviour in the tail region from where one can extract the glueball mass. In comparison, in figure 2 (right), the scaling behaviour of $t_{0}^{2}\left\langle H_{m}\left(x_{0}, t_{0}\right)\right\rangle$ is shown. The difference between these observables decreases as the lattice spacing decreases. The slightly worsened scaling behaviour of the latter for the two relatively larger lattice spacings can be readily attributed to the following. The expression for $H_{m}$ follows directly from the lattice action used in this work, namely, the unimproved Wilson gauge action. On the other hand, the expression for $\bar{E}$ uses the clover definition of the lattice field tensor. Thus the difference in scaling behaviour exhibited in the left and right parts of figure 2 results from the use of an improved versus an unimproved operator, which diminishes as lattice spacing goes to zero. The reason for the non-smooth behaviours of both $\bar{E}$ and $H_{m}$ in case of the ensemble $O_{4}$ is due to the lack of statistics.

In figure 3 we show an example of the effective glueball mass determination from the one-point function. The effective mass $m\left(0^{++}\right)$is plotted versus $x_{0}$ for four different Wilson flow times for the ensemble $\mathrm{O}_{3}$. We find that there is an optimum window of Wilson flow time within which the glueball mass can be reliably extracted from the effective mass plot. For lower values of Wilson flow time, the smearing is not able to remove unwanted contributions completely and the plateau region is too narrow. For higher values of Wilson flow time also plateau is too narrow, presumably due to over smearing resulting in the overlap of the two glueball interpolation operators in the correlation function. Ideally the plateau region should be independent of the flow time chosen, but we observe that an overlapping (within the statistical errors) and extended plateau exists only in the region of flow time between $0.3 \mathrm{fm}$ and $0.35 \mathrm{fm}$. In the following we will present the glueball mass and glueball to vacuum matrix element extracted for these two values of the flow time. 


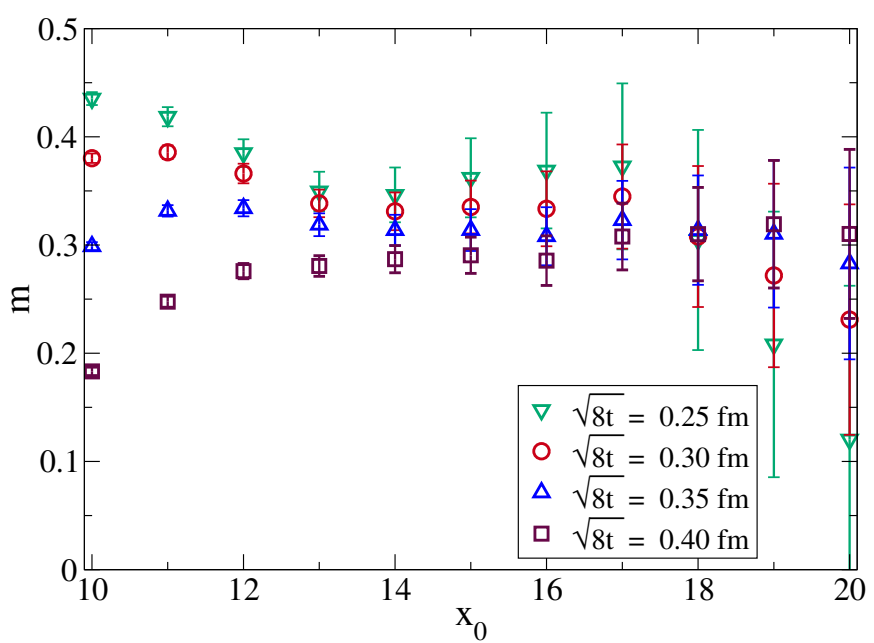

Figure 3. The scalar Glueball effective mass $m\left(0^{++}\right)$as a function of $x_{0}$ extracted from the one-point function of $\bar{E}$ for four values of Wilson flow times for the ensemble $\mathrm{O}_{3}$.

\begin{tabular}{|l|l|l|l|l|l|}
\hline \multirow{2}{*}{ Lattice } & \multirow{2}{*}{ Correlator } & \multicolumn{2}{|c|}{ Mass $m_{G}(\mathrm{MeV})$} & \multicolumn{2}{c|}{ Coefficient $r_{0}^{3} C$} \\
\cline { 3 - 6 } & & $\sqrt{8 t}=0.3 \mathrm{fm}$ & $\sqrt{8 t}=0.35 \mathrm{fm}$ & $\sqrt{8 t}=0.3 \mathrm{fm}$ & $\sqrt{8 t}=0.35 \mathrm{fm}$ \\
\hline \multirow{3}{*}{$O_{1}$} & One point $\left(H_{m}\right)$ & $1729(67)$ & $1649(56)$ & $98(8)$ & $85(6)$ \\
\cline { 2 - 6 } & One point $(\bar{E})$ & $1690(94)$ & $1650(80)$ & $104(14)$ & $95(11)$ \\
\cline { 2 - 6 } & Two point & $1626(186)$ & $1501(96)$ & $103(17)$ & $88(7)$ \\
\hline$P_{1}$ & Two point & $1625(92)$ & $1594(72)$ & $106(10)$ & $100(7)$ \\
\hline \multirow{3}{*}{$O_{2}$} & One point $\left(H_{m}\right)$ & $1700(70)$ & $1645(59)$ & $102(8)$ & $90(6)$ \\
\cline { 2 - 6 } & One point $(\bar{E})$ & $1710(84)$ & $1629(48)$ & $113(12)$ & $97(5)$ \\
\cline { 2 - 6 } & Two point & $1587(234)$ & $1458(115)$ & $101(21)$ & $85(8)$ \\
\hline \multirow{2}{*}{$P_{2}$} & Two point & $1552(61)$ & $1506(71)$ & $99(5)$ & $91(6)$ \\
\hline \multirow{3}{*}{${ }_{3}$} & One point $\left(H_{m}\right)$ & $1640(101)$ & $1551(81)$ & $103(14)$ & $88(9)$ \\
\cline { 2 - 6 } & One point $(\bar{E})$ & $1625(85)$ & $1540(69)$ & $108(12)$ & $93(9)$ \\
\cline { 2 - 6 } & Two point & $1616(254)$ & $1465(161)$ & $99(20)$ & $84(12)$ \\
\hline \multirow{2}{*}{$P_{3}$} & Two point & $1467(181)$ & $1421(182)$ & $94(16)$ & $85(16)$ \\
\hline \multirow{2}{*}{$O_{4}$} & One point $\left(H_{m}\right)$ & $1818(87)$ & $1752(177)$ & $140(13)$ & $125(27)$ \\
\cline { 2 - 6 } & One point $(\bar{E})$ & $1783(141)$ & $1711(273)$ & $144(24)$ & $126(48)$ \\
\cline { 2 - 6 } & Two point & $1521(513)$ & $1459(488)$ & $90(35)$ & $83(33)$ \\
\hline
\end{tabular}

Table 2. The lowest scalar glueball mass $\left(m_{G}\right)$ in $\mathrm{MeV}$ and the glueball to vacuum matrix element in unit of the Sommer parameter $r_{0}$ extracted from correlators at Wilson flow times $\sqrt{8 t}=0.3 \mathrm{fm}$ and $0.35 \mathrm{fm}$. One point and Two point refer to the one point and the two point correlator of $\bar{E}$.

In table 2 we present the values of the lowest scalar glueball mass $m_{G}(=m / a)$ in $\mathrm{MeV}$ and the glueball to vacuum matrix element in unit of the Sommer parameter $r_{0}$ extracted from correlators at Wilson flow times $\sqrt{8 t}=0.3 \mathrm{fm}$ and $0.35 \mathrm{fm}$. One point refers 

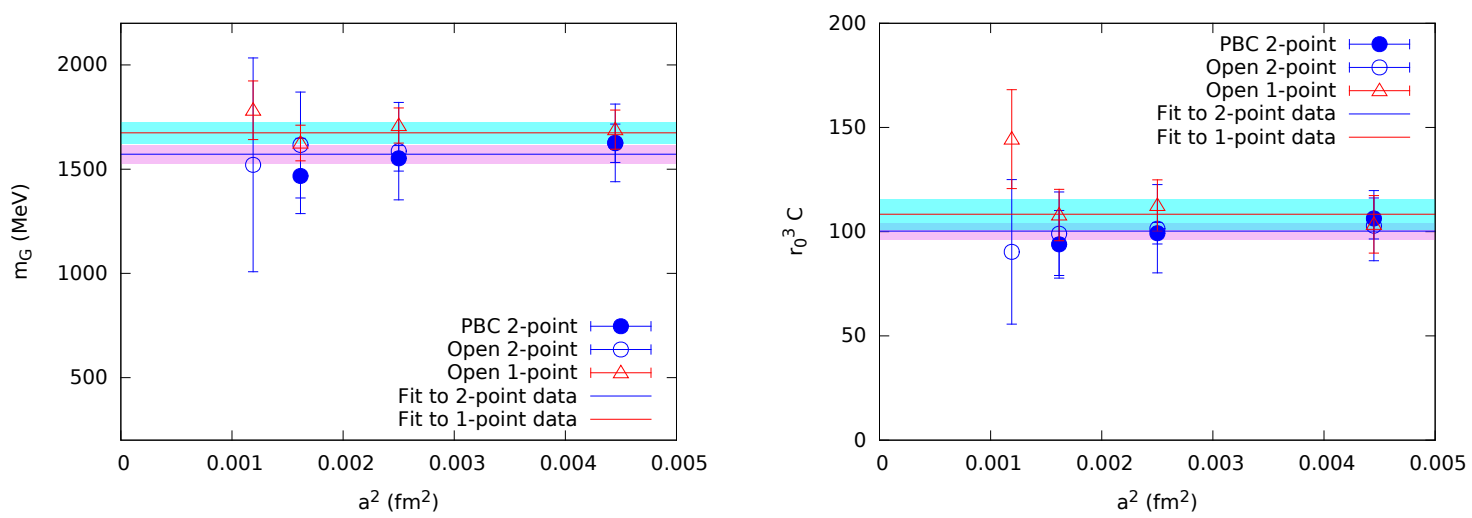

Figure 4. Plot of lowest glueball mass $m_{G}\left(0^{++}\right)$in $\mathrm{MeV}$ (left) and glueball to vacuum matrix element in unit of $r_{0}$ (right) versus $a^{2}$ extracted from one-point (Open) and two-point correlators (Open and PBC) of $\bar{E}$ for different lattice spacings and lattice volumes at Wilson flow time $\sqrt{8 t}=$ $0.3 \mathrm{fm}$. Light blue and light violet shaded regions correspond to the error bands around the fit curves to one-point and two-point data respectively.

to the one point function of $H_{m}$ and $\bar{E}$ and Two point refers to the two point correlator of $\bar{E}$. Wilson (gradient) flow is known [6-8] to have the consequence that the expectation values of local gauge invariant operators constructed from the gauge field at positive flow time are ultraviolet finite. Thus we expect the glueball mass and the glueball to vacuum matrix element extracted at a fixed flow time at different lattice spacings to exhibit scaling (provided lattice artifacts are negligible). The glueball mass should be independent of the flow time but the glueball to vacuum matrix element is expected to depend on the flow time (energy scale). The results presented in table 2 are consistent with these expectations within the statistical errors and the limited range of flow times probed. The investigation of the relationship between the extracted glueball to vacuum matrix element at a given Wilson flow time and its continuum counterpart involves a detailed numerical study of the behaviour of the glueball matrix element of the energy momentum tensor and the trace anomaly under Wilson flow (for related theoretical work, see for example, refs. [17] and [18]) and is beyond the scope of the present work.

In figure 4, we have presented the variation, with $a^{2}$, of lowest glueball mass and glueball to vacuum matrix element in unit of Sommer parameter $r_{0}$ extracted from onepoint (Open) and two-point correlators (Open and PBC) of $\bar{E}$ for different lattice spacings and lattice volumes at Wilson flow time $\sqrt{8 t}=0.3 \mathrm{fm}$. Also shown are separate fits to one-point and two-point data sets. Light blue and light violet shaded regions correspond to the error bands around the fit curves to one-point and two-point data respectively. Corresponding data at Wilson flow time $\sqrt{8 t}=0.35 \mathrm{fm}$ are plotted in figure 5 .

Note that with PBC, glueball observables can be extracted only from the two point correlator. With $\mathrm{PBC}$, the signal can be extracted after performing source averaging and hence the statistical error can be reduced. However, when one simulates at lower lattice spacings it becomes increasingly difficult to generate statistically independent configurations with $\mathrm{PBC}$ in the temporal direction and hence the statistical accuracy begins to 

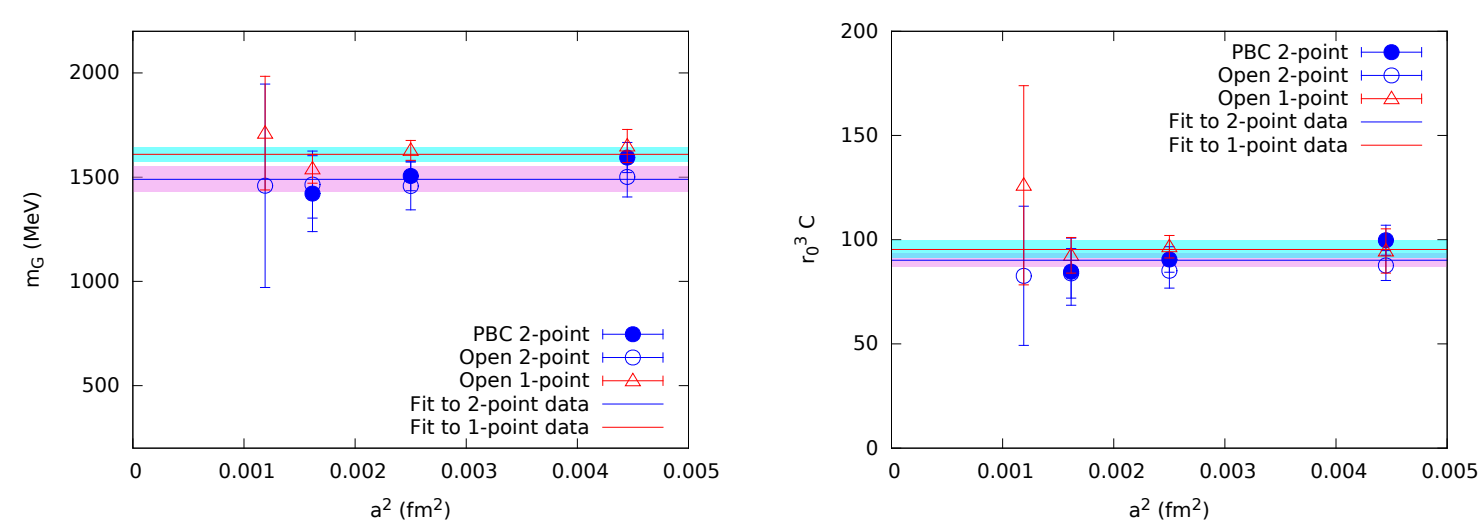

Figure 5. Plot of lowest glueball mass $m_{G}\left(0^{++}\right)$in $\mathrm{MeV}$ (left) and glueball to vacuum matrix element in unit of $r_{0}$ (right) versus $a^{2}$ extracted from one-point (Open) and two-point correlators (Open and PBC) of $\bar{E}$ for different lattice spacings and lattice volumes at Wilson flow time $\sqrt{8 t}=$ $0.35 \mathrm{fm}$. Light blue and light violet shaded regions correspond to the error bands around the fit curves to one-point and two-point data respectively.

\begin{tabular}{|l|l|l|l|l|}
\hline \multirow{2}{*}{ Correlator } & \multicolumn{2}{|c|}{ Mass $m_{G}(\mathrm{MeV})$} & \multicolumn{2}{c|}{ Coefficient $r_{0}^{3} C$} \\
\cline { 2 - 5 } & $\sqrt{8 t}=0.3 \mathrm{fm}$ & $\sqrt{8 t}=0.35 \mathrm{fm}$ & $\sqrt{8 t}=0.3 \mathrm{fm}$ & $\sqrt{8 t}=0.35 \mathrm{fm}$ \\
\hline One point $\left(H_{m}\right)$ & $1701(44)$ & $1628(36)$ & $100(5)$ & $88(4)$ \\
\hline One point $(\bar{E})$ & $1674(51)$ & $1610(35)$ & $108(7)$ & $95(4)$ \\
\hline Two point & $1572(46)$ & $1490(61)$ & $100(4)$ & $90(3)$ \\
\hline
\end{tabular}

Table 3. Fit results for glueball mass and glueball to vacuum matrix element.

suffer. This problem is overcome with OBC in the temporal directions. However in this case translational invariance is lost close to the boundary and hence one can perform source averaging only over the spatial volume and a few temporal slices well inside the bulk. This effectively increases the statistical error. With two point correlator, the disconnected contribution has to be subtracted numerically and hence this contributes to the increase of statistical errors since one is dealing with the subtraction involving two large quantities.

We have shown that, with $\mathrm{OBC}$, the glueball observables can be extracted alternatively from the one point correlation function as well. The automatic subtraction of the disconnected part in the case of one point function leads to smaller statistical error compared to the extraction from two point correlation function with same boundary condition as exhibited in table 2 and figures 4 and 5. One should, of course, keep in mind that this method for the calculation of n-point correlators involving the energy density is applicable only in the scalar channel.

It is noticeable that the results (for both mass and matrix element separately) extracted from the two point correlation functions with periodic and open boundary conditions are very close to each other. However, there is systematically an upward shift in the results obtained from one point correlation functions (doable only with open boundary condition), although not beyond the statistical errors in most cases. We have treated the data sets 
extracted from two point and one point correlation functions separately in the fitting procedure. It is also noteworthy that for the range of lattice spacings explored in this work, scaling violations are within the statistical uncertainty of our data. This led us to perform just constant fits to the data. While fitting the data (both mass and matrix element) for one point correlator, we have excluded $\beta=6.71$ as it somewhat deviates from the general trend. However, we have checked that its inclusion in fitting procedure does not change the results significantly because of large errors on the data at this coupling. The fit results are presented in table 3 .

In our previous work on glueball mass extraction [14], a systematic study of the variation of mass with Wilson flow time was not performed. For each $\beta$, flow time yielding the most stable plateau was picked up. However, for the study of glueball to vacuum matrix element it is mandatory to choose a common flow time for all the lattice spacings. This path has been followed in the present work. This causes the difference in results for glueball mass obtained via the two point correlation functions in this work from that quoted in [14]. However, it is gratifying to note that the average of the glueball masses obtained with the two flow times is very close to the value quoted in [14] and also agrees well with the value given in [19]. Although our results for glueball mass extracted from one point correlation function are comparatively higher, they fall between the results of [19] and [20] both evaluated using two point correlators.

Conclusions. In lattice $\mathrm{QCD}, \mathrm{OBC}$ in the temporal direction has been proposed to overcome the difficulty in the spanning of gauge configurations over different topological sectors. However, the lack of translational invariance in this case can cause some inconveniences in the measurement of observables due to boundary effects when compared to the case of PBC. In this work, we have demonstrated that the same boundary artifacts can be exploited to yield certain observables with greater efficiency. This is achieved by relating the functional average of a generic scalar operator measured in the case of OBC to that with PBC. The scalar glueball mass and the glueball to vacuum matrix elements obtained from this observable in the case of OBC are compared with the values extracted from the measurement of two-point function of the time slice energy density in the case of both PBC and OBC. The Wilson (gradient) flow is used to exhibit the scaling properties of both the mass and the matrix element.

\section{Acknowledgments}

Cray XT5 and Cray XE6 systems supported by the 11th-12th Five Year Plan Projects of the Theory Division, SINP under the Department of Atomic Energy, Govt. of India, are used to perform all the numerical calculations reported in this work. We thank Richard Chang for the prompt maintenance of the systems and the help in data management. We are deeply indebted to Martin Lüscher for the suggestion that glueball mass can be extracted from the expectation value of the energy density in Yang-Mills theory with OBC. This work was in part based on the publicly available lattice gauge theory code openQCD [12]. 


\section{A Connection between matrix elements}

In the following, we show that, with a set of approximations, one can understand why the glueball to vacuum matrix element of both $H_{m}$ and $\bar{E}$ calculated from the one point functions agree (within statistical errors) with that calculated from the two point function of $\bar{E}$.

Consider the two point correlator appearing in eq. (2.11) for the one point function of $\bar{E}\left(x_{0}\right)$. In this correlator, for $x_{0}$, in $\bar{E}\left(x_{0}\right)$, far away from the boundaries the last term in the expression for $\Delta S$ given in eq. (2.3) can be approximated as

$$
\begin{aligned}
\frac{1}{g^{2}} \sum_{\mathbf{x}} \sum_{i} \operatorname{tr}[1- & \left.\operatorname{Re} U_{i 4}(\mathbf{x}, T-1)\right] \\
\approx \frac{1}{g^{2}} \sum_{\mathbf{x}}\{ & \frac{1}{4}\left(\sum_{i} \operatorname{tr}\left[1-\operatorname{Re} U_{i 4}(\mathbf{x}, 0)\right]+\sum_{i} \operatorname{tr}\left[1-\operatorname{Re} U_{i 4}(\mathbf{x}, T-1)\right]\right) \\
& \left.+\frac{1}{4}\left(\sum_{i} \operatorname{tr}\left[1-\operatorname{Re} U_{i 4}(\mathbf{x}, T-1)\right]+\sum_{i} \operatorname{tr}\left[1-\operatorname{Re} U_{i 4}(\mathbf{x}, T-2)\right]\right)\right\} .
\end{aligned}
$$

With this approximation we find, $\Delta S \approx \frac{L^{3}}{2}(\bar{E}(0)+\bar{E}(T-1))$. This leads us to write

$$
\left\langle\bar{E}\left(x_{0}\right)\right\rangle_{\mathrm{OPEN}}=\left\langle\bar{E}\left(x_{0}\right)\right\rangle_{\mathrm{PBC}}+\frac{\left\langle\bar{E}\left(x_{0}\right) e^{\frac{L^{3}}{2}}(\bar{E}(0)+\bar{E}(T-1))\right\rangle_{\mathrm{PBC}}^{\text {connected }}}{\left\langle e^{\frac{L^{3}}{2}(\bar{E}(0)+\bar{E}(T-1))}\right\rangle_{\mathrm{PBC}}} .
$$

Now in continuum limit $\bar{E}\left(x_{0}\right)=a \bar{E}_{Y M}\left(x_{0}\right)$ where $\bar{E}_{Y M}\left(x_{0}\right)=\frac{a^{3}}{2 L^{3}} \sum_{\mathbf{x}} \operatorname{tr}\left\{G_{\mu \nu}(x) G_{\mu \nu}(x)\right\}$. This enables us to write

$$
e^{\frac{L^{3}}{2}(\bar{E}(0)+\bar{E}(T-1))}=1+a \frac{L^{3}}{2}\left(\bar{E}_{Y M}(0)+\bar{E}_{Y M}(T-1)\right)+\mathcal{O}\left(a^{2}\right) .
$$

So eq. (A.1) can be approximated as

$$
\begin{aligned}
& \left\langle\bar{E}\left(x_{0}\right)\right\rangle_{\mathrm{OPEN}} \\
& \quad=\left\langle\bar{E}\left(x_{0}\right)\right\rangle_{\mathrm{PBC}}+a^{2} \frac{L^{3}}{2}\left\langle\bar{E}_{Y M}\left(x_{0}\right)\left(\bar{E}_{Y M}(0)+\bar{E}_{Y M}(T-1)\right)\right\rangle_{\mathrm{PBC}}^{\text {connected }}+\mathcal{O}\left(a^{3}\right) \\
& \quad=\left\langle\bar{E}\left(x_{0}\right)\right\rangle_{\mathrm{PBC}}+C_{1}^{\prime} e^{-m T / 2}\left\{\cosh m\left(\frac{T}{2}-x_{0}\right)+\cosh m\left(\frac{T}{2}-\left(x_{0}+1\right)\right)\right\}+\ldots \\
& \quad \approx\left\langle\bar{E}\left(x_{0}\right)\right\rangle_{\mathrm{PBC}}+2 C_{1}^{\prime} e^{-m T / 2} \cosh m\left(\frac{T}{2}-x_{0}\right)+\ldots
\end{aligned}
$$

As evident from figure $2, H_{m}\left(x_{0}\right)$ can be approximated by $\bar{E}\left(x_{0}\right)$ in the limit of very small lattice spacing. Therefore, in this limit one point function of $H_{m}$ takes the form

$$
\begin{aligned}
\left\langle H_{m}\left(x_{0}\right)\right\rangle_{\mathrm{OPEN}} & =\left\langle H_{m}\left(x_{0}\right)\right\rangle_{\mathrm{PBC}}+\frac{\left\langle H_{m}\left(x_{0}\right) e^{L^{3} H_{m}(T-1)}\right\rangle_{\mathrm{PBC}}^{\text {connected }}}{\left\langle e^{H_{m}(T-1)}\right\rangle_{\mathrm{PBC}}} \\
& \approx\left\langle\bar{E}\left(x_{0}\right)\right\rangle_{\mathrm{PBC}}+\frac{\left\langle\bar{E}\left(x_{0}\right) e^{L^{3} \bar{E}(T-1)}\right\rangle_{\mathrm{PBC}}^{\mathrm{connected}}}{\left\langle e^{L^{3} \bar{E}(T-1)}\right\rangle_{\mathrm{PBC}}} .
\end{aligned}
$$

Discussions subsequent to eq. (A.1) upto eq. (A.3) follow thereafter. 
Open Access. This article is distributed under the terms of the Creative Commons Attribution License (CC-BY 4.0), which permits any use, distribution and reproduction in any medium, provided the original author(s) and source are credited.

\section{References}

[1] M. Lüscher, Topology, the Wilson flow and the HMC algorithm, PoS (LATTICE 2010) 015 [arXiv: 1009.5877] [INSPIRE].

[2] M. Lüscher and S. Schaefer, Lattice QCD without topology barriers, JHEP 07 (2011) 036 [arXiv: 1105.4749] [INSPIRE].

[3] M. Lüscher and S. Schaefer, Lattice QCD with open boundary conditions and twisted-mass reweighting, Comput. Phys. Commun. 184 (2013) 519 [arXiv:1206.2809] [InSPIRE].

[4] S.R. White, Density matrix formulation for quantum renormalization groups, Phys. Rev. Lett. 69 (1992) 2863 [INSPIRE].

[5] N. Shibata and C. Hotta, Boundary effects in the density-matrix renormalization group calculation, Phys. Rev. B 84 (2011) 115116 [arXiv:1106.6202].

[6] M. Lüscher, Trivializing maps, the Wilson flow and the HMC algorithm, Commun. Math. Phys. 293 (2010) 899 [arXiv: 0907.5491] [INSPIRE].

[7] M. Lüscher, Properties and uses of the Wilson flow in lattice QCD, JHEP 08 (2010) 071 [Erratum ibid. 03 (2014) 092] [arXiv: 1006.4518] [INSPIRE].

[8] M. Lüscher and P. Weisz, Perturbative analysis of the gradient flow in non-abelian gauge theories, JHEP 02 (2011) 051 [arXiv:1101.0963] [INSPIRE].

[9] P. Majumdar, N. Mathur and S. Mondal, Noise reduction algorithm for Glueball correlators, Phys. Lett. B 736 (2014) 415 [arXiv:1403.2936] [INSPIRE].

[10] M. Lüscher, Construction of a selfadjoint, strictly positive transfer matrix for euclidean lattice gauge theories, Commun. Math. Phys. 54 (1977) 283 [INSPIRE].

[11] S. Sint, On the Schrödinger functional in QCD, Nucl. Phys. B 421 (1994) 135 [hep-lat/9312079] [INSPIRE].

[12] http://luscher.web.cern.ch/luscher/openQCD/.

[13] A. Chowdhury, A. Harindranath, J. Maiti and P. Majumdar, Topological susceptibility in lattice Yang-Mills theory with open boundary condition, JHEP 02 (2014) 045 [arXiv:1311.6599] [INSPIRE].

[14] A. Chowdhury, A. Harindranath and J. Maiti, Open boundary condition, wilson flow and the scalar glueball mass, JHEP 06 (2014) 067 [arXiv: 1402.7138] [INSPIRE].

[15] A. Chowdhury, A. Harindranath and J. Maiti, Correlation and localization properties of topological charge density and the pseudoscalar glueball mass in $\mathrm{SU}(3)$ lattice Yang-Mills theory, Phys. Rev. D 91 (2015) 074507 [arXiv: 1409.6459] [INSPIRE].

[16] M. Bruno et al., Simulation of QCD with $N_{f}=2+1$ flavors of non-perturbatively improved Wilson fermions, JHEP 02 (2015) 043 [arXiv: 1411.3982] [INSPIRE].

[17] H. Suzuki, Energy-momentum tensor from the Yang-Mills gradient flow, PTEP 2013 (2013) $083 \mathrm{~B} 03$ [Erratum ibid. 2015 (2015) 079201] [arXiv: 1304.0533] [INSPIRE]. 
[18] L. Del Debbio, A. Patella and A. Rago, Space-time symmetries and the Yang-Mills gradient flow, JHEP 11 (2013) 212 [arXiv:1306.1173] [INSPIRE].

[19] H.B. Meyer, Glueball matrix elements: a lattice calculation and applications, JHEP 01 (2009) 071 [arXiv: 0808.3151] [INSPIRE].

[20] Y. Chen et al., Glueball spectrum and matrix elements on anisotropic lattices, Phys. Rev. D 73 (2006) 014516 [hep-lat/0510074] [INSPIRE]. 